\title{
Lutte contre la dégradation des terres agricoles dans le département du Couffo au Bénin
}

\author{
Alfred Bothé Kpadé DOSSA \\ Ministère de l'Economie et des Finances (MEF) et Centre Béninois de la \\ Recherche Scientifique et de l'Innovation (CBRSI), 03 BP 1665 Cotonou, Bénin. \\ E-mail : dossa.alfred@yahoo.fr; Tél : +22997139293/95726000.
}

\section{RESUME}

La dégradation des terres, causée par l'action des eaux et du vent, renforcée par les activités humaines, réduit le rendement et les revenus agricoles et fait baisser la croissance économique notamment dans les pays pauvres comme le Bénin. L'objectif de cette recherche était d'estimer le financement du programme de lutte contre la dégradation des terres dans le département du Couffo, au Sud-Ouest du Bénin. La méthode d'évaluation contingente (MEC) a permis d'obtenir le consentement à payer (CAP) des ménages à l'aide du modèle probit. Ensuite le modèle tobit (Tobin, 1958) est testé par la méthode à deux étapes de Heckman (1979) pour contrôler le biais de sélection de l'échantillon de 267 ménages. Les résultats de l'estimation donnent un CAP moyen de 1557 FCFA / mois / hectare. Pour une campagne agricole de 201739 hectares en 2019, le CAP total est évalué à 3769291476 FCFA/an. Ce montant permettra de lutter contre la dégradation des terres agricoles dans le Couffo au Bénin.

(C) 2020 International Formulae Group. All rights reserved.

Mots clés : Dégradation des terres, modèle de sélection, Couffo, Bénin.

\section{Fighting against agricultural land degradation in the Couffo division in Benin}

\begin{abstract}
Land degradation, caused by the action of water and wind, reinforced by human activities, reduces agricultural yields and incomes and lowers economic growth, especially in poor countries like Benin. The objective of this research was to estimate the funding of the program to combat land degradation in the division of Couffo, in the southwest of Benin. The contingent valuation method (CVM) obtained households' willingness to pay (WTP) using the probit model. Then the tobit (Tobin, 1958) model is tested by Heckman's two-step method (Heckman, 1979) to control the sample selection bias of 267 households. The results of the estimate give an average WTP of 1,557 FCFA / month / hectare. For an agricultural campaign of 201,739 hectares in 2019, the total WTP is estimated at 3,769,291,476 FCFA / year. This amount will help combat the degradation of agricultural land in Couffo, Benin.
\end{abstract}

(C) 2020 International Formulae Group. All rights reserved.

Keywords: Land degradation, selection model, Couffo, Benin. 


\section{INTRODUCTION}

La dégradation des sols constitue un problème environnemental mondial. Elle a une incidence directe sur les moyens de subsistance de millions de personnes, surtout les plus pauvres et les plus vulnérables dans les zones arides de la planète, où plus de 500 millions d'hectares de terres sont dégradés (Fonds pour l'Environnement mondial : FEM, 2014). Elle a un effet négatif sur la capacité d'augmenter la production alimentaire, laquelle est nécessaire pour assurer les besoins alimentaires d'une population qui augmente rapidement. $\mathrm{La}$ dégradation des terres touche 1,5 milliards de personnes et $74 \%$ des populations pauvres dans le monde (Economics of Land Degradation: ELD, 2015). Elle empêche la production agricole et accroit la pauvreté et la vulnérabilité. Elle touche $33 \%$ des terres émergées, affectant plus de 2,6 milliards de personnes dans plus de 100 pays (ELD, 2015). Les coûts financiers et économiques de la dégradation des terres à l'échelle mondiale sont de 3 à $5 \%$ du Produit Intérieur Brut (PIB) agricole pour 42 milliards de dollars US par an et une perte de services écosystémiques de l'ordre de 43400 à 72000 dollars US par km² et entre 870 à 1450 dollars US par personne et par an (ELD, 2015). Au niveau du continent africain, ces coûts varient de 4 à $12 \%$ du PIB, dont $85 \%$ résultant de l'érosion des sols, la perte de nutriments et les changements de cultures (Berry et al., 2003).

Au Bénin, les coûts économiques et financiers de la dégradation des terres varient entre 8,100 et 14,100 milliards de francs CFA et 3 à $5 \%$ du Produit Intérieur Brut (PIB) (Banque Mondiale, 2010). Le département du Couffo est l'une des principales zones de production agricole au sud du Bénin, avec plus de $90 \%$ de sa population dans l'agriculture. Malheureusement, cette zone de très forte pression foncière souffre, contrairement à la théorie de Boserup (1970), de la persistance de l'agriculture minière et de la dégradation prononcée de l'environnement cultural. D'une superficie de $944 \mathrm{~km}^{2}$, le département du Couffo, situé au sud-ouest du Bénin est aussi caractérisé par une forte pression foncière et une dégradation prononcée de l'environnement cultural. En effet, malgré cette pression foncière, la pratique agricole des paysans du département du Couffo demeure essentiellement extractive ; une agriculture sans souci de reconstitution du potentiel de fertilité des sols, c'est-à-dire une agriculture minière. Cet état de choses est contraire à la théorie de Boserup (1970) qui stipule que la densité de la population est un facteur positif pour l'accroissement de la production agricole. En d'autres termes, la pression démographique que connaît cette zone devrait favoriser l'intensification du système de production, l'augmentation de la productivité de la terre et, in fine, le changement technique vers des pratiques agricoles de conservation et d'amélioration des rendements. Ce qui n'est pas le cas. A cet effet, Houngbo et al. (2008), révélaient que plus le producteur dans cette zone est pauvre, moins il met en œuvre les pratiques agricoles de conservation des terres (PAC) telles que la jachère naturelle et les technologies de jachère améliorée à base de Mucuna pruriens et d'Acacia auriculiformis.

L'intérêt tant opérationnel que de la recherche pour la conservation des sols en vue de préserver l'environnement et de lutter contre l'insécurité alimentaire et la pauvreté est croissant. La lutte contre la dégradation des terres agricoles dans le département du Couffo au Bénin est au centre de la présente recherche. Ainsi, l'objectif général de cette recherche est de contribuer à une meilleure conservation des sols. Il s'intéresse donc d'une part, aux déterminants qui influencent le consentement à payer (CAP) des paysans pour la conservation des sols, et d'autre part à l'estimation du montant total qui permettra de lutter efficacement contre la dégradation des terres agricoles dans cette partie du Bénin.

\section{MATERIEL ET METHODES Milieu d'étude}

Le milieu d'étude identifié pour cette recherche est le département du Couffo, regroupant les communes de d'Aplahoué, de Djakotomey, de Dogbo, de Klouékanmey, de Lalo et de Toviklin, et se trouvant dans les zones agro écologiques VI et VII du Bénin. En effet, selon la FAO, une zone agro écologique 
est une unité cartographique de ressources en terres, définie en terme de climat, de géomorphologique et de sols, et/ou de couvert végétal et possédant un éventail spécifique de potentiels et de contraintes pour l'utilisation des terres. La zone agro écologique VI comprend les communes de Djakotomey, Dogbo, Klouékanmey et Toviklin en plus d'autres communes et est caractérisée en raison des caractéristiques de ses sols zone de « terre de barre ». Le climat est marqué par deux (02) saisons de pluies (mars-juillet; octobrenovembre) et deux (02) saisons sèches (décembre-février; août). Les hauteurs d'eau de pluie varient entre 1000 à $1400 \mathrm{~mm}$. Quant à la zone agro écologique VII, elle comprend la commune de Lalo et plusieurs autres communes. Elle est dénommée zone de dépression. C'est la plus petite des huit (08) zones agro écologiques en termes de superficie. Sur le plan climatique, elle est assez comparable à la zone des terres de barre avec cependant une forte humidité relative. Dans les deux (02) zones agro écologiques, on y cultive du maïs, du manioc, du niébé, de l'arachide, du piment et de la tomate.

\section{Population d'étude : l'échantillonnage}

L'approche terrain a permis de recueillir des informations auprès des ménages agricoles. Ainsi, sur la base de critères rigoureux, il sera procédé à un échantillonnage aléatoire par commune. Ce sont les données de l'Institut National de la Statistique et de l'Analyse Economique (INSAE, 2015) qui ont aidé à la discrimination entre les villages. Par rapport aux groupes cibles, à la méthodologie utilisée et aux phénomènes à étudier, seuls les chefs de ménage ont été interrogés. La formule de Schwartz (1995) permettra de calculer la taille de l'échantillon (n) :

$\mathrm{n}=\frac{\mathrm{t}^{2} \times \mathrm{p}(1-\mathrm{p})}{\mathrm{E}^{2}}$, où

$\mathrm{n}=$ taille de l'échantillon

$\mathrm{t}=$ degré de confiance à $95 \%$ (valeur type de $1,96)$

$\mathrm{p}=$ estimation de la proportion de la population spécifique concernée par l'étude. Lorsqu'elle n'est pas définie, elle est égale à $50 \%$ ).
$\mathrm{E}=$ marge d'erreur tolérée : jusqu'à $6 \%$. Ainsi : $\mathrm{n}=\frac{\mathrm{t}^{2} \times \mathrm{p}(1-\mathrm{p})}{\mathrm{E}^{2}}=\frac{1,96^{2} \times 0,5(1-0,5)}{0,06^{2}}$, d'où $\mathrm{n}=$ 267

La structure de l'échantillon des ménages par sexe est présenté dans le Tableau 1

\section{Modèle d'étude}

L'outil de collecte des données est le questionnaire contingent. La Méthode d'Evaluation Contingente (MEC) a permis d'obtenir empiriquement le consentement à payer (CAP) des ménages bénéficiaires pour éviter la dégradation de leurs terres. En estimant le CAP moyen à l'aide du modèle probit, le modèle tobit (Tobin, 1958) est testé par la méthode à deux (02) étapes de Heckman (1979) pour contrôler le biais de sélection de l'échantillon de 267 ménages. Deux (02) situations $\mathrm{S} 1$ et S2 sont proposées aux enquêtés:

- S1 : garder la situation actuelle de dégradation des terres. Cette situation n'entraîne aucune charge financière à l'endroit des ménages, mais ils vont continuer de subir les nuisances liées à la dégradation et l'infertilité actuelles des sols agricoles de leur commune.

- $\boldsymbol{S 2}$ : participer financièrement au programme d'amélioration de la qualité des sols. Cette situation entraîne un coût financier pour les ménages, mais leur permet d'éviter la baisse de fertilité des sols qu'ils subissent actuellement.

Dans une première étape, l'individu décide ou non de payer pour les techniques de conservation des sols (TCS). Cette décision peut être représentée par un modèle qualitatif dichotomique basée sur un certain critère $y_{1, i}^{*}$.

$\left\{\begin{array}{l}\text { Si } y_{1, i}^{*}>0, \text { l'individu i décide de payer } \\ \text { Si } y_{1, i}^{*} \leq 0, \text { l'individu i décide de ne pas payer }\end{array}\right.$

Dans une seconde étape, l'individu décide du montant qu'il va consacrer aux TCS, après avoir décidé de payer. On a alors un modèle de données censurées puisque, si l'on note $y_{2, i}$ le paiement effectif du ménage i, celuici est défini par $\forall \mathrm{i}=1,2, \ldots, \mathrm{N}$ : 


$$
y_{2, i}=\left\{\begin{array}{c}
y_{2, i}^{*} \text { Si } y_{1, i}^{*}>0 \\
0 \text { Si } y_{1, i}^{*} \leq 0
\end{array}\right.
$$

Le modèle économétrique utilisé pour estimer le CAP des enquêtés, relève du domaine des variables qualitatives, plus précisément des modèles de sélection. En effet, les variables que nous cherchons à expliquer sont les montants de CAP déclarés par les ménages pour contribuer à l'amélioration de la qualité des terres agricoles dans le département du Couffo. Or, il est certain que ces informations ne sont disponibles que pour les ménages ayant optés pour S2. Il s'agit d'un modèle à deux étapes. Dans un premier temps, le ménage choisit de participer au programme ou non, ensuite il décide du montant à payer. En utilisant la méthode de Heckman (1979), notre modèle peut se formaliser mathématiquement comme suit pour chaque ménage i :

- Equation de sélection : participer au programme d'amélioration de la qualité des sols. Soit Z, la variable qualitative, tel que $\mathrm{Z}=$ 1 si le ménage i participe au programme et 0 sinon :

$Z=w_{i} \beta+\mu_{i} . \quad \mu_{i}$ suit une loi normale de paramètres $\mathrm{N}(0,1)$.

- Equation substantielle : estimation du consentement à payer (CAP) annoncé (observable uniquement si $\mathrm{Z}=1$ ) :

$Y=x_{i} \alpha_{i}+\varepsilon_{i}$

$\boldsymbol{\varepsilon}_{i}$ suit une loi normale de paramètres $\mathrm{N}$ $(0,1)$.

Les $w_{i}$ et $x_{i}$ sont des variables socioéconomiques observables. En admettant une loi normale $\mathrm{N}(0,1)$, les termes d'erreur des deux équations (de sélection et substantielle), sont donc absolument continues et admettent pour densité $\mathrm{f}(\mathrm{x})$, telle que :

$$
\mathrm{f}(\mathrm{x})=\frac{1}{\sqrt{2 \pi}} \mathrm{e}^{-\mathrm{x}^{2} / 2}, \text { avec } \mathrm{x}=\mu_{i}, \varepsilon_{i} \text {. }
$$

Ce genre de modèle est normalement estimé par la Méthode du Maximum de Vraisemblance (MMV). Cependant, comme la convergence s'avère parfois difficile, l'estimateur de Heckman (1979), obtenu en deux étapes, lui est parfois préféré. L'équation de sélection est d'abord estimée par un modèle
Probit, ensuite une régression par les Moindres Carrés Ordinaires (MCO) permet d'obtenir les coefficients de la deuxième équation. La méthode de Heckman (1979) permet ainsi une approximation des résultats trouvés par la MMV. Pour l'équation de sélection, la personne interrogée répond oui si l'utilité qu'il associe à la conservation des sols excède son utilité actuelle (dégradation et baisse de fertilité des sols) et non sinon. Il s'agit là d'un cas polaire ou ambivalent. Il sera donc utilisé un modèle à choix binaire (Greene, 2003). La variable à expliquer est alors dite qualitative à deux modalités (oui $=1$ et non $=0$ ). Le niveau d'utilité des personnes interrogées correspondant à chacune des deux réponses n'est pas connu. Il s'agit d'un modèle à utilité aléatoire. Il est supposé que la fonction d'utilité se compose de deux parties : une partie déterministe qui dépend d'un ensemble de caractéristiques (sexe, âge, niveau de revenu, sensibilité par rapport à la qualité de l'environnement) des personnes interrogées et une partie aléatoire qui correspond à la composante des préférences individuelles connues des personnes interrogées, mais inconnues du chercheur. L'existence de cette composante aléatoire oblige à raisonner en termes de probabilité : comment caractériser la probabilité pour qu'une personne accepte de payer le montant proposé, étant données ses caractéristiques individuelles ? La probabilité de répondre « oui » correspond donc à la probabilité pour qu'une personne estime qu'elle est dans une meilleure situation avec le scénario proposé, même en contribuant financièrement.

Notre équation de sélection s'inspire des travaux de Mäler (1974). Considérons le programme primal d'une personne enquêtée qui maximise sa fonction d'utilité $U$ pour un vecteur de prix $\mathrm{P}$ et un niveau de revenu $\mathrm{Y}$ donné. Son programme s'écrit de la façon suivante :

$\operatorname{Max} U(X, Q)$, sous contrainte de $: P X=Y$

Ce programme admet une solution qui est une fonction d'utilité indirecte définie par :

$u_{i j}=u_{i}\left(p, y_{j}, q^{i}, m_{j}\right)$ 
Avec $\mathrm{j}$ un ménage enquêté donné, et $\mathrm{i}=$ 1 correspond à la qualité de l'environnement après conservation des terres agricoles et $\mathrm{i}=0$ au statu quo (la situation actuelle). $P$, le vecteur de prix, le revenu de $\mathrm{j}$ est noté $\mathrm{y}_{\mathrm{j}} ; \mathrm{m}_{\mathrm{j}}$ représente un vecteur de caractéristiques de l'individu (sexe, âge,...), et $\mathrm{q}$ la qualité de l'environnement qui dépend de la qualité des sols agricoles. Pour une amélioration de la qualité de l'environnement entre les situations 0 et 1 correspondant à une amélioration de la qualité des sols qui passe de $\mathrm{q}^{0}$ à $\mathrm{q}^{1}$ (avec $\mathrm{q}^{1}>$ $\left.\mathrm{q}^{0}\right)$, on assistera à une amélioration des rendements de production agricole due à une amélioration de la fertilité des sols. L'utilité du paysan augmente en passant de $u_{0}$ à $u_{1}$. On a : $u_{l j}=u_{l}\left(p, y_{j}, q^{l}, m_{j}\right)$

La mesure du bien-être du ménage due à une modification de la qualité des sols est le surplus compensateur $(\mathrm{SC}=\Omega)$. Soit : $\Omega=u_{l j}-u_{0 j}=u_{1}\left(p, y_{j}, q^{l}, m_{j}\right)-u_{0}\left(p, y_{j}, q^{0}\right.$, $\left.m_{j}\right)$

Pour disposer d'une meilleure qualité des sols $\left(q^{1}\right)$ afin d'éviter les dégradations des terres et les baisses de fertilité, le ménage serait prêt à participer financièrement au programme en réduisant sa consommation de biens privés au profit des biens environnementaux (qualité des sols agricoles). L'équation du CAP d'un ménage agricole $\mathrm{j}$ à estimer sera donc :

$C A P_{j}\left(q^{l}-q^{0}\right)=f\left(m_{j}, u_{o}, q^{I}-q^{0}\right)+\mu_{j}$

Avec $\mu_{\mathrm{j}}$ la composante aléatoire des préférences inconnues du ménage agricole enquêté.

Puisque la composante aléatoire des préférences est inconnue, on peut seulement faire des hypothèses probabilistes sur les réponses « oui » et « non ». On note $\mathrm{Yj}$ la réponse à ces questions : $\mathrm{Yj}=1$ correspond à la réponse « oui » c'est-à-dire accepte de contribuer financièrement au programme. $\mathrm{La}$ probabilité de répondre oui est donc :

$\operatorname{Pr}\left(Y_{j}=1\right)=\operatorname{Pr}\left(u_{1}\left(y_{j}-C A P_{j}, m_{j} q^{l}, \mu_{l j}\right) \geq u_{0}\right.$ $\left.\left(y_{j}-C A P_{j}, m_{j} q^{0}, \mu_{0 j}\right)\right)$

Dans ce modèle à utilité aléatoire linéaire, la fonction d'utilité se présente sous une forme additivement séparable. Elle se décompose ainsi en une partie déterministe linéaire $\left(v_{i}\right)$ et une partie stochastique $\left(\mu_{i j}\right)$. $u_{j}\left(y_{j}, m_{j} \mu_{i j}\right)=v_{j}\left(y_{j}, m_{j}\right)+\mu_{i j} ; \operatorname{avec} v_{j}\left(y_{j}, m_{j}\right)$ $=m_{j} \alpha+y_{j} \beta$

On a donc :

$\operatorname{Pr}\left(Y_{j}=1\right)=\operatorname{Pr}\left(v_{1}\left(y_{j}-C A P_{j}, m_{j}, \mu_{l j}\right) \geq v_{0}\left(y_{j}\right.\right.$, $\left.\left.m_{j}, \mu_{0_{j}}\right)\right)$

$=\operatorname{Pr}\left(v_{l}\left(y_{j}-C A P_{j}, m_{j}, \mu_{l j}\right)-v_{0}\left(y_{j}, m_{j}, \mu_{0 j}\right) \geq 0\right)$

La fonction d'utilité indirecte est spécifiée comme la somme d'une composante déterministe et d'une composante aléatoire. La différence des deux composantes aléatoires peut être identifiée en un unique terme d'erreur tel que :

$\mu_{j}=\mu_{l j}-\mu_{0 j}$

Dans la partie déterministe de la fonction d'utilité indirecte, on a :

$v_{l j-} v_{0 j}=m_{j}\left(\alpha_{l}-\alpha_{0}\right)+\left(y_{j-} C A P_{j}\right) \beta_{l}-y_{j} \beta_{0}$ (16)

On fait l'hypothèse que l'utilité marginale du revenu est constante entre les deux états. Il en résulte que $\beta_{1}=\beta_{0}=\beta$, et en notant que $\alpha=\alpha_{1}-\alpha_{0}$, la probabilité de répondre « oui » est alors donnée par l'équation suivante

$\operatorname{Pr}(Y j=1)=\operatorname{Pr}\left(m_{j} \alpha . C A P_{j} \beta+\mu_{j}\right)>0$

On suppose que les termes aléatoires $\mu_{i j}$ sont indépendants et identiquement distribués selon la même loi, avec une espérance nulle. Deux lois sont possibles pour les $\mu j$ : la loi normale et la loi logistique. Lorsqu'on utilise la loi normale centrée réduite, on parle de modèle Probit et lorsqu'on utilise la loi logistique, on parle du modèle Logit (Mignon, 2008).

- Le modèle Probit est celui pour lequel F est la fonction de répartition de la loi normale centrée réduite :

$F(w)=\Phi(w)=\int_{-\infty}^{w} \frac{1}{\sqrt{2 \pi}} e^{-\frac{t^{2}}{2}} d t$

Ce qui donne :

$P(Y=1)=\int_{-\infty}^{X \beta} \frac{1}{\sqrt{2 \pi}} e^{-\frac{t^{2}}{2}} d t=\Phi(X \beta)$

- Le modèle Logit est celui pour lequel F est la fonction de répartition de la loi logistique :

$F(w)=L(W)=\frac{e^{w}}{1+e^{w}}=\frac{1}{1+e^{-w}}$

Ce qui donne :

$$
P(Y=1)=\frac{1}{1+\exp (-X \beta)}=L(X \beta)
$$


L (fonction de répartition de la loi logistique) et $\Phi$ (fonction de répartition de la loi normale centrée réduite) sont toutes les deux symétriques par rapport à 0 , et comprises entre 0 et 1 (ce qui convient parfaitement pour représenter une probabilité). La loi logistique de fonction de répartition $\mathrm{L}$ a pour moyenne 0 et pour variance $\frac{\pi^{2}}{3}$. Il est donc naturel de comparer à $\Phi(\mathrm{w})$, fonction de répartition de $\mathrm{N}$ $(0,1)$, la fonction $\mathrm{L}_{1}(\mathrm{w})$ où :

$\mathrm{L}_{1}(\mathrm{w})=\frac{1}{1+\exp (-\pi w / \sqrt{3})}$

Dans la plupart des cas, on peut indifféremment choisir l'un ou l'autre modèle, car ces lois sont proches l'une de l'autre. Le modèle Logit a l'avantage d'une plus grande simplicité numérique. Le modèle Probit est en revanche plus proche du modèle habituel de régression par les Moindres Carrés Ordinaires (MCO). Toutefois, le choix entre les deux modèles pour les résidus de la régression latente est véritablement arbitraire. Cependant, ce choix conduit en général à des estimateurs différents des paramètres. Quel modèle choisir? Pour les départager, on peut tester les deux modèles. Tester le logit contre le probit, revient à tester $\alpha=1$ contre $\alpha=0$. Pour ce faire, on utilise un test de score qui a l'avantage : (1) de ne pas nécessiter d'estimer le modèle général (on estime le modèle sous l'hypothèse nulle seulement), (2) d'avoir une distribution standard malgré le fait que le test se fasse à la frontière du domaine (puisque $0 \leq \alpha \leq 1$ ). Il y a donc très peu de différence entre les paramètres estimés $(\hat{\beta})$ sous les deux modèles. Marpsat et Trognon (1992) ont montré que les estimateurs obtenus avec le modèle Logit sont $\frac{\pi}{\sqrt{3}}$ fois plus grands environs que ceux obtenus par le modèle Probit; soit $\hat{\beta}_{\text {logit }} \approx 1,8 \hat{\beta}_{\text {probit. }}$ Quant à Amemiya (1981), il propose en première approximation d'utiliser la relation $\hat{\beta}_{\text {logit }} \approx 1,6 \hat{\beta}_{\text {probit }}$ entre les estimations logit et probit. Par contre, d'autres auteurs soutiennent qu'il n'y a pas de tests économétriques pour choisir entre les deux modèles logit et probit (Hoetker, 2007). Dans la pratique, les résultats des deux méthodes sont similaires et ces deux méthodes sont indifféremment choisies
(Marpsat et Trognon, 1992), sauf sur de très grands échantillons (Williams, 2009).

Si $\mu j$ suit une loi normale $N\left(\boldsymbol{0}, \boldsymbol{\sigma}^{2}\right), \quad \Theta j=\frac{\mu j}{\sigma}$ suit une loi centrée réduite. On a alors :

$\operatorname{Pr}(Y j=1)=\operatorname{Pr}\left(\mu j<m_{j} \alpha-C A P_{j} \beta\right)$

$=\operatorname{Pr}\left(\Theta j<m_{j} \frac{\alpha}{\sigma}-C A P_{j} \frac{\beta}{\sigma}\right)$

$=\phi\left(m_{j} \frac{\alpha}{\sigma}-C A P_{j} \frac{\beta}{\sigma}\right)$

Si $\mu j$ suit une loi logistique de moyenne nulle et de variance $\frac{\pi^{2} \sigma_{L}{ }^{2}}{3}$, la probabilité pour que $j$ réponde « oui » est :

$\operatorname{Pr}(Y j=1)=\frac{1}{1+\exp \left[-\left(m_{j} \frac{\alpha}{\sigma_{L}}-C_{L} P_{j} \frac{\beta}{\sigma}\right)\right]}$

Pour calculer alors ${ }^{L}$ le consentement à payer (CAP) pour un modèle à utilité aléatoire, on le définit théoriquement comme une somme d'argent qui laisse la personne interrogée indifférente entre le statu quo et la situation proposée. Le CAP est ainsi défini par :

$\mathrm{m}_{\mathrm{j}} \alpha_{1}+\left(y_{j}-C A P_{\mathrm{j}}\right) \beta+\mu_{l j}=\mathrm{m}_{\mathrm{j}} \alpha_{0}+y_{j} \beta+\mu_{0 j}$; et le CAP du ménage j est donc :

$C A P_{j}=m_{j} \frac{\alpha}{\beta}+\frac{\mu j}{\beta}$

Pour calculer le CAP moyen E(CAP) sur l'échantillon, la formule est la suivante :

$\mathrm{E}(\mathrm{CAP})=\bar{m} \frac{(\alpha / \sigma)}{(\beta / \sigma)}$

Avec $\bar{m}$, le vecteur des moyennes des caractéristiques individuelles du ménage. Le coefficient des variables de la matrice $m$ correspond à l'estimation $\frac{\alpha}{\sigma}$ et le coefficient associé au montant proposé correspond à une estimation de $\frac{\beta}{\sigma}$ (en fait, les modèles logit ou probit fournissent une estimation de $-\beta / \sigma$ ).

L'analyse de la distribution du CAP, montre théoriquement qu'il est compris entre 0 et le revenu de la personne interrogée. Comment alors s'assurer de ce cadrage en pratique ? Autrement dit comment «borner» le consentement à payer ? Haab et McConnell (2005) proposent un modèle qui borne correctement le CAP.

Ce modèle s'écrit :

$C A P j=G(m j \gamma+\mu j) y_{j}$; où $0 \leq G(m j \gamma+$

$\mu j) \leq 1$ et $G(m j \gamma+\mu j) \geq 0$

La fonction $\mathrm{G}$ exprime le consentement à payer comme une fraction du revenu. La version la plus utilisée de ce modèle est : 


$$
C A P j=\frac{y_{j}}{1+\exp \left(-m_{j} \gamma-\mu_{j}\right)}
$$

Supposons que le terme d'erreur suit une loi normale, le modèle devient alors :

$\operatorname{Pr}(Y j=1)=\varnothing\left(\frac{m_{j} \gamma+\log \frac{y_{j}-C A P_{j}}{C A P_{j}}}{\sigma}\right)$

Par contre si le terme d'erreur suit une loi logistique, alors le modèle s'écrit :

$$
\operatorname{Pr}(Y j=1)=\frac{1}{1+\exp \left(-\frac{m_{j} \gamma+\log \frac{y_{j}-C A P_{j}}{{ }^{\prime} A P_{j}}}{\sigma}\right)}
$$

Avec le vecteur des termes «montant/revenu »: $\log \frac{y_{j}-C A P_{j}}{C A P j}$.
Le coefficient des variables de la matrice $m$ correspond à l'estimation $\frac{\gamma}{\sigma}$ et le coefficient associé à la variable «montant/revenu » correspond à une estimation de $\frac{1}{\sigma}$.

L'enquête dans le département du Couffo, nous a permis de catégoriser six (06) variables de l'équation de sélection et quatre (04) variables de l'équation substantielle avec la prédiction de leurs signes (Tableau 2).

\begin{tabular}{|c|c|c|c|c|c|c|}
\hline \multirow{2}{*}{ Communes } & \multirow{2}{*}{$\begin{array}{c}\text { Population } \\
\text { totale } \\
\text { (RGPH4) }\end{array}$} & \multirow{2}{*}{$\begin{array}{c}\text { Nombre de } \\
\text { ménages }\end{array}$} & \multirow{2}{*}{$\begin{array}{l}\text { Taille de } \\
\text { ménage }\end{array}$} & \multirow{2}{*}{$\begin{array}{l}\text { Nombre de } \\
\text { ménages tiré } \\
\text { par commune }\end{array}$} & \multicolumn{2}{|c|}{ Sexe } \\
\hline & & & & & Masculin & Féminin \\
\hline APLAHOUE & 171109 & $\begin{array}{l}33100 \\
(25 \%)\end{array}$ & 5,2 & $\begin{array}{c}67 \\
(25 \%)\end{array}$ & $58(25 \%)$ & $\begin{array}{c}9 \\
(25 \%)\end{array}$ \\
\hline DJAKOTOMEY & 134028 & $\begin{array}{l}25106 \\
(18 \%)\end{array}$ & 5,3 & $\begin{array}{c}48 \\
(18 \%)\end{array}$ & $42(18 \%)$ & $\begin{array}{c}6 \\
(18 \%)\end{array}$ \\
\hline DOGBO & 103057 & $\begin{array}{l}21199 \\
(15 \%)\end{array}$ & 4,9 & $\begin{array}{c}40 \\
(15 \%)\end{array}$ & $35(15)$ & $\begin{array}{c}5 \\
(15 \%)\end{array}$ \\
\hline KLOUKANMEY & 128597 & $\begin{array}{l}25531 \\
(18 \%)\end{array}$ & 5,0 & $\begin{array}{c}48 \\
(18 \%)\end{array}$ & $42(18 \%)$ & $\begin{array}{c}6 \\
(18 \%)\end{array}$ \\
\hline LALO & 119926 & $\begin{array}{l}17735 \\
(12 \%)\end{array}$ & 6,8 & $\begin{array}{c}32 \\
(12 \%)\end{array}$ & $28(12 \%)$ & $\begin{array}{c}4 \\
(12 \%)\end{array}$ \\
\hline TOVIKLIN & 88611 & $\begin{array}{l}17773 \\
(12 \%)\end{array}$ & 5,0 & $\begin{array}{c}32 \\
(12 \%)\end{array}$ & $28(12 \%)$ & $\begin{array}{c}4 \\
(12 \%)\end{array}$ \\
\hline Total & 745328 & $\begin{array}{l}140444 \\
(100 \%)\end{array}$ & 5,3 & $267(100 \%)$ & $\begin{array}{c}233 \\
(87 \%)\end{array}$ & $\begin{array}{c}34 \\
(13 \%)\end{array}$ \\
\hline
\end{tabular}

Tableau 1 : Structure de l'échantillon des ménages par sexe.

Source : Calculé à partir des statistiques de l'INSAE (2015).

Tableau 2 : Variables entrant dans les équations de sélection et substantielle du modèle.

\begin{tabular}{llll}
\hline $\begin{array}{c}\text { Variables de l'équation de sélection et } \\
\text { prévision de leur signe }\end{array}$ & \multicolumn{2}{c}{$\begin{array}{c}\text { Variables de l'équation substantielle et } \\
\text { prévision de leur signe }\end{array}$} \\
\hline \multicolumn{1}{c}{ Variables } & Signes & Variables & Signes \\
\hline Revenu agricole (REVENU) & + & Age du paysan (AGE) & + \\
\hline Revenu non agricole (NONAG) & + & Taille du ménage (TM) & + \\
\hline Taille du ménage (TM) & + & Accès au marché (ACCMAR) & + \\
\hline Age du paysan (AGE) & + & Revenu agricole (REVENU) & + \\
\hline Niveau d'éducation (EDU) & + & & \\
\hline Accès au marché (ACCMAR) & + & & \\
\hline \multicolumn{2}{c}{ Source : Auteur, 2020. }
\end{tabular}

Source : Auteur, 2020 


\section{RESULTATS}

La variable dépendante qui est le consentement à payer (CAP) pour la lutte contre la dégradation des terres dans le Couffo, est dichotomique. Elle définit si oui ou non le ménage consent à payer pour les mesures de conservation des sols. $\mathrm{CAP}=1$, si le ménage consent à payer ; CAP $=0$ sinon. C'est une variable expliquée du modèle probit. Quant aux variables indépendantes, elles sont de deux (02) catégories : les variables économiques (la taille du ménage, le revenu agricole, le revenu non agricole et l'accès au marché) et les variables sociales (le niveau d'éducation du chef de ménage et son âge). Ce sont des variables explicatives censées influencer le CAP. Les résultats de l'enquête dans le département du Couffo présentent 80 chefs de ménages, soit $30 \%$ qui ont choisi $\mathrm{S}_{1}\left(y_{1, i}^{*} \leq 0\right.$, donc ayant un CAP $=0$ ), et $70 \%$ ayant fait l'option $\mathrm{S}_{2}\left(y_{1, i}^{*}>0\right.$, c'est-à-dire qui accepte le programme de lutte contre la dégradation des sols agricoles, représentant ainsi 187 ménages ayant effectivement accepté d'investir dans la conservation des sols). Ainsi, sur les 187 ménages, 80 consentent payer 1500 FCFA, tandis que 55 ménages acceptent de payer 2500 FCFA. Les ménages agricoles qui acceptent de payer 3000 FCFA sont 36 et ceux qui consentent payer 3500 FCFA sont au total 14 ménages. La dernière catégorie de ménages ayant accepté d'investir pour un montant de 4000 FCFA dans les techniques de conservation des sols dans le Couffo, comprend seulement 2 ménages et se trouve dans la commune d'APLAHOUE. Les CAP moyens des six (06) communes du Couffo ayant accepté le programme (S2) et pour tout l'échantillon des 267 ménages (S1+S2) sont présentés dans le Tableau 3.

La moyenne des CAP moyens des six (06) communes pour S2 est alors égale à 2225,34 FCFA et pour ( $1+$ S2), elle est égale à 1556,47 FCFA. Pour la première étape, l'estimation de l'équation de sélection est portée sur la totalité des observations ayant répondu au scénario contingent, donc sur un échantillon de 267 ménages agricoles. Pour la deuxième étape relative à l'équation substantielle, STATA utilise les observations de $S_{2}$, ceux qui ont décidé de payer. Cette estimation porte sur 187 observations. A partir du modèle étudié, les coefficients de l'équation de sélection s'interprètent comme ceux du modèle probit. Quant aux coefficients de l'équation substantielle, ils représentent l'influence de la variable explicative sur la variable expliquée. Il s'agit d'un moindre carré ordinaire (MCO). La Figure 1 affiche les consentements à payer moyen de ceux qui acceptent le programme de lutte contre la dégradation des terres agricoles (187 chefs de ménage) et de tout l'échantillon des 267 chefs de ménage dans le milieu d'étude.

Pour la significativité des coefficients des variables entrant dans les équations de sélection et substantielle du modèle, il faut noter que pour le modèle de sélection (première étape), les coefficients des variables accès au marché (ACCMAR) et âge du paysan (AGE) ne sont pas significatifs. Quant à l'équation substantielle (deuxième étape), c'est l'âge du paysan qui n'a aucune influence sur le montant $\mathrm{du}$ consentement à payer. Par contre, les coefficients des variables «taille du ménage » (TM) et « revenu agricole » (REVENU), sont significatifs à $1 \%$ aussi bien à la $1^{\text {ère }}$ qu'à la $2^{\text {ème }}$ étape. Les résultats de l'estimation à deux étapes de Heckman (1979) sont présentés dans le Tableau 4.

Le CAP moyen pour les ménages qui acceptent participer au programme de lutte contre la dégradation des terres étant égale à 1556,47 FCFA/mois /hectare pour l'ensemble de l'échantillon. Les superficies totales (ST) emblavées au cours des périodes 2018 et 2019 étant respectivement égales à 167396 hectares et 201739 hectares, il en résulte un CAP total (CAPT) de 3127626864 FCFA/an en 2018 et 3769291476 FCFA/an pour 2019 dans tout le département du Couffo. Le détail des CAP moyens communaux pour les périodes 2018 et 2019 sont présentés dans le Tableau 5.

Le Tableau 5 montre que les CAP totaux sont une fonction croissante des superficies totales emblavées. Autrement dit, plus grande est la superficie emblavée, plus grand est le CAP total. 
Tableau 3 : Détermination des CAP moyens des 6 communes du Couffo.

$\begin{array}{cccccc}\mathrm{S} 1+\mathrm{S} 2 & y_{1, i}^{*} \leq 0 & \text { CAP } & y_{1, i}^{*}>0 & \text { CAP } & \text { CAP } \\ & (\mathrm{S} 1) & (\mathrm{S} 1) & (\mathrm{S} 2) & (\mathrm{S} 2) & (\mathrm{S} 1+\mathrm{S} 2)\end{array}$

\begin{tabular}{lllllll}
\hline Aplahoue & 67 & 20 & 0 & 47 & 2351 & 1649 \\
\hline Djakotomey & 48 & 14 & 0 & 34 & 2471 & 1750 \\
\hline Dogbo & 40 & 12 & 0 & 28 & 2339 & 1638 \\
\hline Kloukanmey & 48 & 14 & 0 & 34 & 2191 & 1552 \\
\hline Lalo & 32 & 10 & 0 & 22 & 2114 & 1453 \\
\hline Toviklin & 32 & 10 & 0 & 22 & 1886 & 1297 \\
\hline Total & 267 & 80 & - & 187 & - & -
\end{tabular}

Source : Auteur, 2020.

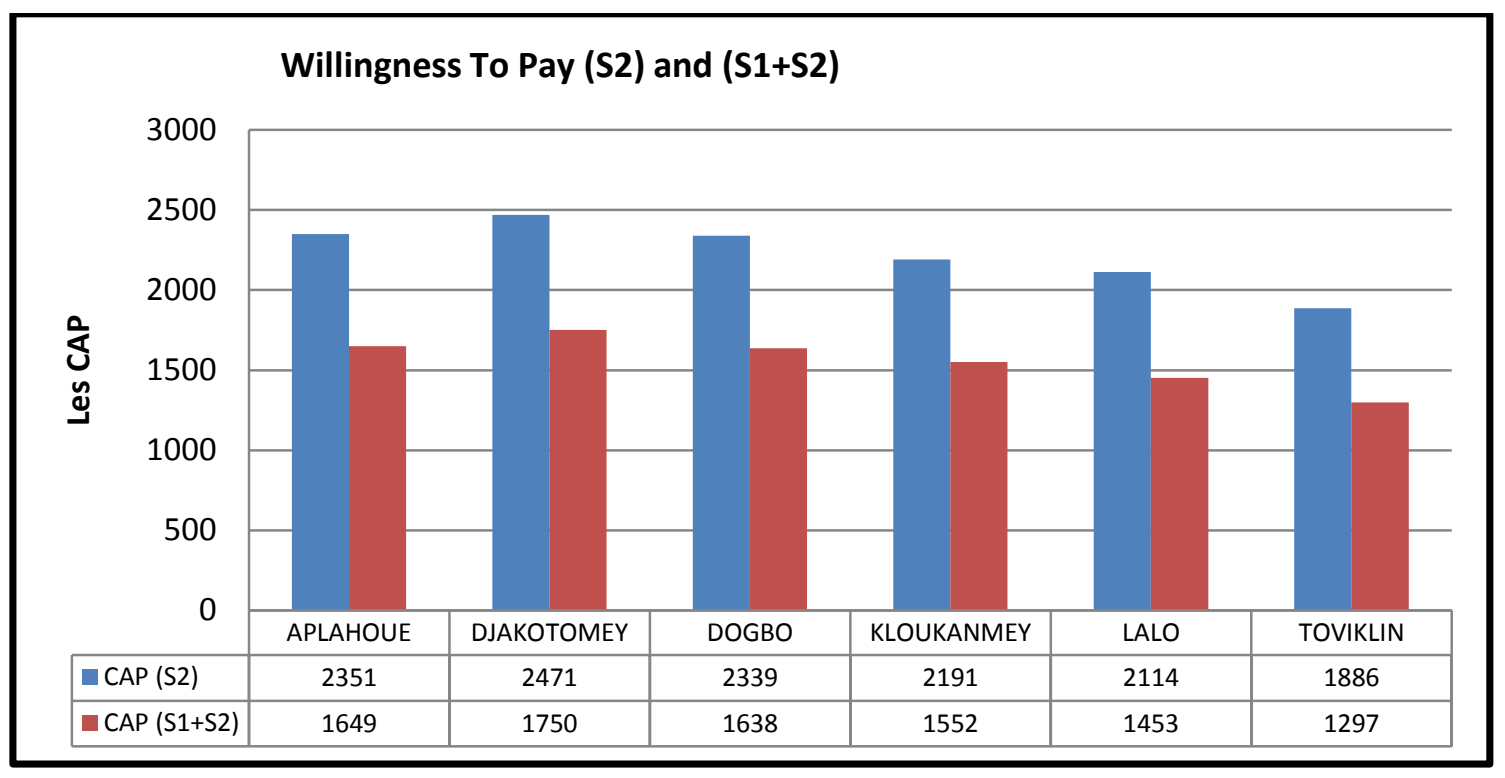

Figure 1 : Les consentements à payer moyen de ceux qui acceptent investir et de tout l'échantillon. Source : Auteur, 2020.

Tableau 4 : Résultats de l'estimation à deux étapes de Heckman (1979).

\begin{tabular}{ccccc}
\hline \multicolumn{7}{c}{$\mathbf{1}^{\text {ère }}$ étape $:$ Equation de sélection } & Consentement à payer - CAP) \\
\hline Variables & Coefficients & $\mathbf{z}$ & $\mathbf{p}>|\mathbf{z}|$ & Significativité des coefficients \\
\hline TM & $0,2282053^{* * *}$ & 6,56 & 0,000 & $1 \%$ \\
\hline REVENU & $-5,56 \mathrm{e}-06^{* * *}$ & $-2,61$ & 0,009 & $1 \%$ \\
\hline NONAG & $1,689863^{* * *}$ & 5,42 & 0,000 & $1 \%$ \\
\hline ACCMAR & $-0,2657782$ & $-0,70$ & 0,483 & Non significatif \\
\hline AGE & $-0,0096559$ & $-0,95$ & 0,343 & Non significatif \\
\hline EDUC & $0,6898728^{* *}$ & 2,55 & 0,011 & $5 \%$
\end{tabular}




\begin{tabular}{ccccc}
\hline cons & 0,6247104 & 0,84 & 0,403 & Non significatif \\
\hline $\mathbf{2}^{\text {ème }}$ étape $:$ Equation substantielle & (Montant & du consentement à payer $-M C A P)$ \\
\hline Variables & Coefficients & $\mathrm{t}$ & $\mathrm{p}>|\mathrm{t}|$ & Significativité des coefficients \\
\hline TM & $-115,0171^{* * *}$ & $-10,89$ & 0,000 & $1 \%$ \\
\hline REVENU & $0,0032173^{* * *}$ & 4,24 & 0,000 & $1 \%$ \\
\hline ACCMAR & $-552,5768^{* * *}$ & $-4,67$ & 0,000 & $1 \%$ \\
\hline AGE & 11,32648 & 0,80 & 0,425 & Non significatif \\
\hline Mills_ & $-119,8337$ & $-0,76$ & 0,451 & Non significatif \\
\hline Cons & $3132,75^{* * *}$ & 10,20 & 0,000 & $1 \%$ \\
\hline \multicolumn{7}{c}{ Source: Réalisé à partir des régressions Probit et MCO dans STATA, 2020. }
\end{tabular}

Tableau 5 : Détermination des CAP annuels communaux et totaux.

\begin{tabular}{|c|c|c|c|c|c|}
\hline & \multirow{2}{*}{$\begin{array}{c}\text { CAP moyens } \\
\text { annuels } \\
\text { communaux }\end{array}$} & \multicolumn{2}{|c|}{$\begin{array}{l}\text { Superficies totales } \\
\text { emblavées (hectare) }\end{array}$} & \multicolumn{2}{|c|}{ CAP Totaux (FCFA) } \\
\hline & & 2018 & 2019 & 2018 & 2019 \\
\hline Aplahoue & 19788 & 50056 & 64180 & 990508128 & 1269993840 \\
\hline Djakotomey & 21000 & 35101 & 33228 & 737121000 & 697788000 \\
\hline Dogbo & 19656 & 14750 & 21038 & 289926000 & 413522928 \\
\hline Klouekanmey & 18624 & 34487 & 43326 & 642285888 & 806903424 \\
\hline Lalo & 17436 & 18330 & 23567 & 319601880 & 410914212 \\
\hline Toviklin & 15564 & 14672 & 16400 & 228355008 & 255249600 \\
\hline $\begin{array}{l}\text { Département } \\
\text { du Couffo }\end{array}$ & 18684 & 167396 & 201739 & 3127626864 & 3769291476 \\
\hline
\end{tabular}

Source : A partir des statistiques de la Direction Départementale de l'Agriculture, de l'Elevage et de la Pêche du Couffo (DDAEP, 2020).

\section{DISCUSSION}

De toutes les variables censées influencer la décision des paysans d'adopter les techniques de conservation des sols (cultures de couvertures, fossé de déviation, paillage, billons, jachère minimum, cultures sur les courbes de niveau, pas de brûlis, bandes enherbées, fossés, haies, terrasses, composts, fumure organique, cultures mixtes moins érosives, digues, agroforesterie), les facteurs économiques semblent de loin être les plus importants. Ainsi, la taille du ménage (TM) se réfère au nombre d'individus dans le ménage. Dans cette étude, les ménages à grande taille (comprise entre 8 et 10 personnes) sont plus disponibles à investir dans la conservation du sol. C'est une variable explicative quantitative. Il était attendu un signe positif $(+)$ de cette variable. Ceci est confirmé par de nombreux travaux empiriques (Kpondenou et al., 2019 ; Asrat et al., 2004). Par contre, la $2^{\text {ème }}$ étape de l'estimation de Heckman (1979), enregistre un signe négatif (-). Il est à noter que les paysans les plus riches sont plus disposés à pratiquer les techniques de conservation. Il est démontré que la superficie totale de champ emblavée est positivement corrélée au revenu. Le revenu agricole (REVENU) est une variable explicative quantitative. Il était attendu un signe positif $(+)$ du coefficient de cette variable aux deux (02) étapes (Probit et MCO). Mais les résultats des enquêtes sur le terrain donnent un

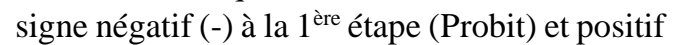
$(+)$ pour la $2^{\text {ème }}$ étape $(\mathrm{MCO})$. Ceci est confirmé par de nombreuses études empiriques en Afrique de l'ouest (Dossa, 2016 ; Kouadio 
et al., 2018). Quant au revenu non agricole (NONAG), il indique si le ménage bénéficie d'une source de revenu non agricole, à part le revenu agricole ou pas. Elle prend la valeur 1 si le ménage perçoit un revenu non agricole et 0 sinon. C'est une variable explicative qualitative. Il était attendu dans cette étude un signe positif $(+)$ de cette variable sur le CAP. Les résultats des enquêtes sur le terrain ont confirmé ce signe à la $1^{\text {ère }}$ étape du modèle probit. La variable accès au marché (ACCMAR) a pour proxy dans cette étude, la qualité du déplacement du champ au marché. Ainsi, il a été pris en compte sur le terrain le moyen de transport disponible, la distance du village du fermier au marché le plus proche et l'état de la piste qui mène à cette route. ACCMAR prend la valeur 1 si le village du paysan est plus proche du marché et 0 autrement. Plus le transport est relativement commode, plus il est supposé que le fermier est désenclavé et a donc accès au marché. Les travaux de recherche de Houngbo et al., (2008) ont montré que cette variable influence positivement le CAP des paysans pour la pratique des mesures de conservation des sols. C'est une variable explicative qualitative. Dans cette étude, il été attendu un signe positif (+) de cette variable. Malheureusement, les résultats des enquêtes sur le terrain donnent un signe

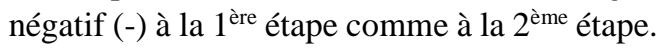

Bien que les facteurs économiques soient certainement importants dans la décision d'investir pour adopter des pratiques de conservation, ils ne tiennent pas compte de tous les comportements d'adoption. Les facteurs sociaux ou sociologiques sont également importants et, pour certains agriculteurs, éleveurs et propriétaires fonciers, peuvent même l'emporter sur les considérations économiques (Adimassu et al., 2015). L'importance des variables sociales a également été soulignée par le travail de plusieurs chercheurs (Asrat et al., 2004; Ilboudo et al., 2020) qui ont offert des explications sur la croissance économique du tiers monde et sur la protection de l'environnement à travers l'analyse des caractéristiques sociales de la personnalité. Il existe plusieurs facteurs sociologiques qui influencent les paysans à investir dans les techniques de conservation des sols. Dans le cadre de l'enquête dans le département du Couffo, il est retenu globalement deux (02) variables sociales : le niveau d'éducation du chef de ménage (EDU) et l'âge du paysan (AGE). Le niveau d'éducation du chef de ménage (EDU) est une variable dummy, qui prend la valeur 1 si le chef de ménage est éduqué et 0 sinon. L'éducation accroît les potentialités du paysan à recevoir, comprendre et utiliser les informations. C'est une variable explicative qualitative. Il est supposé avoir un effet positif sur la décision de payer pour les pratiques de conservation des sols. Les résultats des enquêtes sur le terrain ont confirmé ce signe à la $1^{\text {ère }}$ étape du modèle probit. Quant à la variable âge du chef du ménage (AGE), elle mesure l'âge du chef de famille en nombre d'années. C'est pour capter l'effet de l'âge sur le CAP pour les mesures de conservation des sols. On peut introduire la variable âge au carré $\left(\mathrm{AGE}^{2}\right)$ dans le modèle, permettant ainsi de prendre en compte l'indétermination de l'effet de l'âge démontrée par la théorie économique, dû aux différences d'horizons temporels selon l'âge et de ses implications (Houngbo et al., 2008). C'est une variable explicative quantitative. Dans notre étude il était attendu un effet positif (+) de l'âge sur le CAP. Mais, les résultats des enquêtes sur le terrain donnent un signe négatif (-) à la $1^{\text {ère }}$ étape et positif (+) à la $2^{\text {ème }}$ étape.

Par ailleurs, le modèle de sélection à deux (02) étapes de Heckman (1979) estime l'inverse de ratio de Mills. L'inverse du ratio de Mills n'étant pas significatif, il y a donc absence de biais de sélection. Le modèle choisit pour cette étude est donc bien spécifié et bien calibré. Cependant, on note une significativité de $1 \%$ de la constance (_cons) au niveau de la régression des moindres carrés ordinaires (MCO) de la $2^{\text {ème }}$ étape, ceci montre qu'il y a des variables pertinentes qui sont absentes dans la régression MCO.

\section{Conclusion}

Les travaux d'enquête dans le département du Couffo ont abouti à deux (02) catégories de résultats : les résultats descriptifs, 
issus de l'enquête sur les déterminants du consentement à payer (CAP) et les résultats économétriques de l'estimation à 2 étapes de Heckman (1979). Ces travaux ont permis d'exprimer en grandeur monétaire ce que les ménages agricoles des six (06) communes du Couffo sont prêts à payer pour une amélioration de la qualité de leurs sols afin d'éviter les baisses de fertilité, pouvant provoquer des risques d'insécurité alimentaire et de pauvreté. Les résultats obtenus ont permis de tirer des conclusions aussi bien méthodologiques, qu'analytiques et pratiques. Il est constaté que les ménages agricoles dans le département du Couffo accordent une importance à l'amélioration de la qualité de leurs sols et sont prêts à y contribuer financièrement. Cette étude $a$, en outre, mis en évidence l'influence significative des variables socio-économiques sur la décision des paysans à payer pour l'amélioration de la qualité de leurs sols. Les valeurs calculées, constitueront des références de base pour les recherches futures visant l'appréhension de la valeur économique des sols agricoles de façon générale et permettront d'éclairer les décideurs publics en matière de politique agricole au Bénin.

\section{CONFLITS D'INTERETS}

L'auteur de ce papier déclare qu'il n'y a aucun conflit d'intérêts. Il a pleinement et totalement contribué à son élaboration et à sa rédaction.

\section{CONTRIBUTIONS DES AUTEURS}

ABKD a conçu, préparé et a mis en œuvre la collecte, l'analyse des données et la rédaction du papier.

\section{REMERCIEMENTS}

L'auteur remercie les ménages enquêtés (les chefs de famille) pendant la phase de la collecte des données pour leurs oreilles attentives qu'ils ont prêtées aux questions à eux posées. Il remercie également les élus locaux des différentes communes du département du Couffo pour lui avoir accordé leur disponibilité. Enfin, la Direction Départementale de l'Agriculture, de l'Elevage et de la Pêche (DDAEP) du Couffo pour la mise à disposition des données quantitatives.

\section{REFERENCES}

Adimassu Z, Langan S, Johnston R. 2015. Comprendre les déterminants des investissements des agriculteurs dans des pratiques durables de gestion des terres en Éthiopie : examen et synthèse. Environnement, Développement et Durabilité, 18 : 1005-1023. DOI : 10.1007/s10668-015-9683-5

Amemiya T. 1975. Qualitative Response Models: A Survey. Journal of Economic Literature, 19(4): 1483-1536. DOI: https://www.jstor.org/stable/2724565

Asrat P, Belay K, Hamito D. 2004. Determinants of farmer's willingness to pay for soil conservation practices in the southeastern Highlands of Ethiopia. Land Degradation and Development, 15(4): 423-438. DOI : https://doi.org/10.1002/ldr.623

Banque Mondiale. 2010. Analyse environnementale, République du Bénin. Département du Développement Durable. Rapport $\mathrm{N}^{\circ}$ 58190-BJ, Région Afrique, Washington DC, 1-70.

Berry L, Olson J, Campbell D. 2003. Assessing The Extent, Cost and Impact of Land Degradation at The National Level: Findings and Lessons Learned from Seven Pilot Case Studies (Chapter 1). Commissioned by Global Mechanism with support from the World Bank. 1-18. https://www.researchgate.net/publication 1272682048

Boserup E, 1970. Evolution Agraire et Pression Démographique. Flammarion : Paris.

Dossa ABK. 2016. Evaluation et financement des dépenses environnementales : cas du consentement à payer pour la conservation des sols agricoles à Kérou au Bénin. Thèse de Doctorat, Université d'Abomey-Calavi, Bénin ; p. 207.

Economics of Land Degradation - ELD. 2015. La valeur des terres. Terres prospères et résultats positifs grâce à une gestion durable des terres. Rapport principal de 
l'ELD, septembre 2015. Disponible sur www.eld-initiative.org

Fonds pour l'Environnement mondial- FEM. 2014. Combattre la dégradation des sols dans les zones d'activité économique. www.theGEF.org,

Greene HW. 2003. Econometric Analysis (5 ${ }^{\text {th }}$ Edition, Chapters 5-9). Pearson Education International. New York University.

Haab T, McConnell K. 2005. Valuing Environmental and Natural Resources: the Econometrics of Non-Market Valuation. American Journal of Agricultural Economics, 87(2): 529-530. DOI: https://doi.org/10.1111/j.00029092.2005.740_2.x

Heckman J. 1979. Sample Selection Bias as a Specification Error, Econometrica, 47(1): 153-162.

DOI:

https://www.jstor.org/preview-

page $/ 10.2307 / 1912352$ ?seq $=1$

Hoetker G. 2007. The Use of Logit and Probit Models in Strategic Management Research. Strategic Management Journal 28(4): 331-343. DOI: https://www.onlinelibrary.wiley.com/doi /abs/10.1002/smj.582

Houngbo NE, Sinsin B, Floquet A. 2008. Pauvreté chronique et pauvreté transitoire sur le plateau Adja au Bénin: caractéristiques et influence sur la mise en œuvre des pratiques agricoles de conservation des terres. Tropicultura, 26(3) : $\quad 186-189 . \quad$ DOI: www.tropicultura.org/text/v26n3/186.pd $\mathrm{f}$

Ilboudo A, Soulama S, Hien E, Zombre P. 2020. Perceptions paysannes de la dégradation des ressources naturelles des bas-fonds en zone soudano-sahélienne : cas du sous bassin versant du NakanbéDem au Burkina Faso. Int. J. Biol. Chem. Sci., 14(3): 883-895. DOI: 10.4314/ijbcs.v14i3.19.

Institut National des Statistiques et de l'Analyse Economique - INSAE. 2015.
Quatrième Recensement Général de la Population et de l'Habitation (RGPH4) : Que retenir des effectifs de population en 2013 ? MDAEP/Bénin.

Kouadio YJ-C, Soiret KSP, Kpan WB, Yao NO, N'Guessan KE, Kouassi KP et Gomez PJ. 2018. Valeur de conservation de la Réserve Naturelle Volontaire (RNV) de la Dodo, Sud-Ouest de la Côte d'Ivoire (Afrique de l'Ouest). Int. J. Biol. Chem. Sci., 12(6) : 2784-2796. DOI: 10.4314/ijbcs.v12i6.24

Kpadenou CC, Tama C, Tossou BD, Yabi JA. 2019. Déterminants socio-économiques de l'adoption des pratiques agroécologiques en production maraîchère dans la vallée du Niger au Bénin. Int. J. Biol. Chem. Sci., 13(7): 3103-3118. DOI: https://dx.doi.org/10.4314/ijbcs.v13i7.11

Mäler KG. 1974. Environmental Economics: A Theoretical Inquiry John Hopkins (chapter 6). University Press : Baltimore, MD.

Marpsat M, Trognon A. 1992. Présentation générale du modèle Logit (chapitres 910). Journées de méthodologie statistique. INSEE Méthodes $\mathrm{N}^{\circ} 46-47$ 48.

Mignon V. 2008. Econométrie: Théorie et Applications. Economica : Paris, France.

Schwartz D. 1995. Méthodes Statistiques à l'Usage des Médecins et des Biologistes ( $4^{\mathrm{ème}}$ edition). Editions médicales Flammarion: Paris.

Tobin J. 1958. Estimation of Relationships for Limited Dependent Variables. Econometrica, 26(1): 24-36. DOI: $10.2307 / 1907382$

Williams R. 2009. Using Heterogeneous Choice Models to Compare Logit and Probit Coefficients Across Groups. Sociological Methods \& Research, 37: 531-559.

DOI: https://doi.org/10.1177\%2F00491241093 35735 . 